PROCEEDINGS OF THE

AMERICAN MATHEMATICAL SOCIETY

Volume 137, Number 3, March 2009, Pages 869-875

S 0002-9939(08)09566-X

Article electronically published on September 15, 2008

\title{
THE ACTION OF THE HEAT OPERATOR ON JACOBI FORMS
}

\author{
OLAV K. RICHTER
}

(Communicated by Ken Ono)

\begin{abstract}
We investigate the action of the heat operator on Jacobi forms. In particular, we present two explicit characterizations of this action on Jacobi forms of index 1. Furthermore, we study congruences and filtrations of Jacobi forms. As an application, we determine when an analog of Atkin's $U$-operator applied to a Jacobi form is nonzero modulo a prime.
\end{abstract}

\section{INTRODUCTION}

The Ramanujan theta operator $\Theta:=q \frac{d}{d q}=\frac{1}{2 \pi i} \frac{d}{d \tau}$ plays an important role in the theory of modular forms. If $f$ is a modular form of weight $k$, then it is easy to see that

$$
\Theta(f)=\frac{k}{12} f E_{2}+\hat{f},
$$

where $E_{2}$ is the "quasimodular" Eisenstein series of weight 2 and $\hat{f}$ is a modular form of weight $k+2$. In 1916, Ramanujan [14] noticed the identities

$$
\Theta\left(E_{4}\right)=\frac{1}{3}\left(E_{4} E_{2}-E_{6}\right) \quad \text { and } \quad \Theta\left(E_{6}\right)=\frac{1}{2}\left(E_{6} E_{2}-E_{8}\right),
$$

where $E_{4}, E_{6}$, and $E_{8}$ are the usual (modular) Eisenstein series of weights 4,6 , and 8 , respectively. However, for an arbitrary modular form $f$, an explicit description of $\hat{f}$ in Equation (11) was not known until 2004 when Bruiner, Ono, and Kohnen [3] established an exact formula.

In this paper, we consider an analogous situation for Jacobi forms. The heat operator $L_{m}:=\frac{1}{(2 \pi i)^{2}}\left(8 \pi i m \frac{\partial}{\partial \tau}-\frac{\partial^{2}}{\partial z^{2}}\right)$ is a natural tool in the study of Jacobi forms (see, for example, $\S 3$ of Eichler and Zagier 7] and Choie [4, [5], among others). If $\phi$ is a Jacobi form of weight $k$ and index $m$, then a direct computation reveals that

$$
L_{m}(\phi)=\frac{(2 k-1) m}{6} \phi E_{2}+\hat{\phi}
$$

where $E_{2}$ is again the "quasimodular" Eisenstein series of weight 2 and $\hat{\phi}$ is a Jacobi form of weight $k+2$ and index $m$. This observation justifies the following two remarks:

a) If $p \geq 5$ is a prime, then $E_{2}$ is a $p$-adic modular form of weight 2 in the sense of Serre [15]. Hence Equation (3) implies that if $\phi$ is a Jacobi form of weight $k$ and

Received by the editors March 5, 2008

2000 Mathematics Subject Classification. Primary 11F50; Secondary 11F60.

(C)2008 American Mathematical Society 
index $m$, then $L_{m}(\phi)$ is a $p$-adic Jacobi form of weight $k+2$ and index $m$ in the sense of Guerzhoy [9].

b) The Ramanujan theta operator preserves the ring of quasimodular forms (see Kaneko and Zagier [11]). Equation (31) shows that the heat operator preserves the ring of quasi-Jacobi forms (in the sense of Kawai and Yoshioka [12]).

In this paper, we take a closer look at the action of $L:=L_{1}$ on Jacobi forms of weight $k$ and index 1 . Section 2 gives two different explicit characterizations of $\hat{\phi}$ in Equation (3) when $m=1$. In particular, we have the following generalizations of the Ramanujan identities (2):

$$
L\left(E_{4,1}\right)=\frac{7}{6}\left(E_{4,1} E_{2}-E_{6,1}\right) \quad \text { and } \quad L\left(E_{6,1}\right)=\frac{11}{6}\left(E_{6,1} E_{2}-E_{8,1}\right),
$$

where $E_{4,1}, E_{6,1}$, and $E_{8,1}$ denote the Jacobi Eisenstein series of index 1 and weights 4,6 , and 8 , respectively.

In Section 3, we investigate congruences of Jacobi forms. We prove two results on filtrations of Jacobi forms, which allow us to generalize work of Ahlgren and Ono [1]. More specifically, we define an analog of Atkin's $U$-operator for Jacobi forms and we determine when that operator applied to a Jacobi form is nonzero modulo a prime.

\section{EXPLICIT DESCRIPTION OF THE ACTION OF $L$}

We introduce some standard notation. Let $\mathbb{Z}$ and $\mathbb{N}$ denote the sets of integers and nonnegative integers, respectively, and let $\mathbb{H} \subset \mathbb{C}$ be the complex upper half plane. Set $\Gamma:=\mathrm{SL}_{2}(\mathbb{Z}), \Gamma_{0}(N):=\left\{\left(\begin{array}{ll}a & b \\ c & d\end{array}\right) \in \Gamma \mid c \equiv 0(\bmod N)\right\}$, and let $M_{k}\left(\Gamma_{0}(N)\right)$ be the vector space of modular forms of weight $k$ on $\Gamma_{0}(N)$. If $N=1$, then we write $M_{k}:=M_{k}\left(\Gamma_{0}(1)\right)$. Now let us recall the definition of a Jacobi form (for more details, see [7]).

Definition 1. A Jacobi form of weight $k$ and index $m(k, m \in \mathbb{N})$ on $\Gamma$ is a holomorphic function $\phi: \mathbb{H} \times \mathbb{C} \rightarrow \mathbb{C}$ satisfying the transformation laws

$$
\phi\left(\frac{a \tau+b}{c \tau+d}, \frac{z}{c \tau+d}\right)=(c \tau+d)^{k} e^{2 \pi i m \frac{c z^{2}}{c \tau+d}} \phi(\tau, z), \text { for all }\left(\begin{array}{ll}
a & b \\
c & d
\end{array}\right) \in \Gamma,
$$

and

$$
\phi(\tau, z+\lambda \tau+\mu)=e^{-2 \pi i m\left(\lambda^{2} \tau+2 \lambda z\right)} \phi(\tau, z), \text { for all }(\lambda, \mu) \in \mathbb{Z}^{2} .
$$

Furthermore, one requires that a Jacobi form has a Fourier expansion of the form

$$
\phi(\tau, z)=\sum_{\substack{n, r \in \mathbb{Z} \\ 4 n m-r^{2} \geq 0}} c(n, r) q^{n} \zeta^{r},
$$

where here (and in the following) $q:=e^{2 \pi i \tau}$ and $\zeta:=e^{2 \pi i z}(\tau \in \mathbb{H}, z \in \mathbb{C}$ ). A Jacobi form that satisfies the stronger condition $c(n, r) \neq 0 \Rightarrow 4 n m>r^{2}$ is called a cusp form. We denote the vector space of Jacobi forms of weight $k$ and index $m$ by $J_{k, m}$ and the space of Jacobi cusp forms of weight $k$ and index $m$ by $J_{k, m}^{\text {cusp }}$.

Basic examples of Jacobi forms are given by the Jacobi-Eisenstein series $E_{k, m}$ (see $\S 2$ of [7]). In particular, if $m=1$, then one has the following Fourier expansions:

$$
\begin{aligned}
& E_{4,1}(\tau, z)=1+\left(\zeta^{2}+56 \zeta+126+56 \zeta^{-1}+\zeta^{-2}\right) q+\ldots \\
& E_{6,1}(\tau, z)=1+\left(\zeta^{2}-88 \zeta-330-88 \zeta^{-1}+\zeta^{-2}\right) q+\ldots \\
& E_{8,1}(\tau, z)=1+\left(\zeta^{2}+56 \zeta+366+56 \zeta^{-1}+\zeta^{-2}\right) q+\ldots
\end{aligned}
$$


Apply $L$ to the Fourier expansions of $E_{4,1}$ and $E_{6,1}$, observe that

$$
E_{2}(\tau)=1-24 q+\ldots,
$$

and note that $J_{6,1}$ and $J_{8,1}$ are one-dimensional to verify that the generalizations of the Ramanujan identities in (4) hold.

Let $\phi$ be an arbitrary Jacobi form of weight $k$ and index 1 . We seek an explicit formula for the Jacobi form $\hat{\phi}$ in Equation (3). Note that there exist unique $f \in M_{k-4}$ and $g \in M_{k-6}$ (see Theorem 3.5 in [7]) such that $\phi=f E_{4,1}+g E_{6,1}$. As an immediate consequence of the identities in (4), we obtain the following characterization of $\hat{\phi}$ in Equation (3).

Theorem 1. If $\phi=f E_{4,1}+g E_{6,1} \in J_{k, 1}\left(f \in M_{k-4}, g \in M_{k-6}\right)$, then

$$
L(\phi)=\frac{2 k-1}{6} \phi E_{2}+\left(4 \hat{f}-\frac{11}{6} g E_{4}\right) E_{4,1}+\left(4 \hat{g}-\frac{7}{6} f\right) E_{6,1},
$$

where $\hat{f}$ and $\hat{g}$ are defined by Equation (1) and explicitly determined in [3].

Alternatively, we can also use the results in Atkinson 2 to describe $\hat{\phi}$ in Equation (3) in terms of its theta decomposition. We give this description in the next theorem, after introducing necessary notation. For $\mu=0,1$, set

$$
\theta_{1, \mu}(\tau, z):=\sum_{\substack{r \in \mathbb{Z} \\ r \equiv \mu(\bmod 2)}} q^{\frac{r^{2}}{4}} \zeta^{r} .
$$

If $\phi \in J_{k, 1}$, then

$$
\phi(\tau, z)=h_{0}(\tau) \theta_{1,0}(\tau, z)+h_{1}(\tau) \theta_{1,1}(\tau, z)
$$

where

$$
h_{0}(\tau)=\sum_{N=N_{0}}^{\infty} c_{0}(N) q^{N} \quad \text { and } \quad h_{1}(\tau)=q^{-\frac{1}{4}} \sum_{N=N_{1}}^{\infty} c_{1}(N) q^{N}
$$

are, loosely speaking, modular forms of weights $k-\frac{1}{2}$ (for details, see $\S 5$ of [7]). Note that if $\phi \not \equiv 0$, then there exist minimal $N_{0}, N_{1} \in \mathbb{N}$ such that $C_{0}\left(N_{0}\right) \neq 0 \neq C_{1}\left(N_{1}\right)$. Set $M:=\min \left(4 N_{0}, 4 N_{1}-1\right)$ and set

$$
\alpha_{h_{0}}:=-\frac{5}{6}\left(k-\frac{1}{2}\right)+2 N_{0}+2 M, \quad \beta_{h_{0}}:=\frac{2}{3}\left(k-\frac{1}{2}\right)-\frac{8}{3} N_{0}-\frac{4}{3} M,
$$

and

$$
\alpha_{h_{1}}:=-\frac{5}{6}\left(k-\frac{1}{2}\right)+2 N_{1}+2 M, \quad \beta_{h_{1}}:=\frac{2}{3}\left(k-\frac{1}{2}\right)-\frac{8}{3} N_{1}-\frac{4}{3} M .
$$

Finally, let $\mathcal{F}$ be a fundamental domain for $\Gamma_{0}(4)$, let $H_{\tau^{\prime}}(\tau)$ be the (meromorphic) modular form of weight 2 discussed in detail in [2], and let

$$
\left(h_{\mu}\right)_{\theta}:=\sum_{\tau^{\prime} \in \mathcal{F}} \operatorname{ord}_{\tau^{\prime}}\left(h_{\mu}\right) H_{\tau^{\prime}}
$$

Now we are in a position to characterize $\hat{\phi}$ in Equation (3) in terms of its theta decomposition. 
Theorem 2. Let $\phi(\tau, z)=h_{0}(\tau) \theta_{1,0}(\tau, z)+h_{1}(\tau) \theta_{1,1}(\tau, z) \in J_{k, 1}$. Then

$$
L(\phi)=\frac{2 k-1}{6} \phi E_{2}-4 \sum_{\mu=0}^{1} h_{\mu}\left(\left(h_{\mu}\right)_{\theta}+\alpha_{h_{\mu}} E_{2}^{(2)}+\beta_{h_{\mu}} E_{2}^{(4)}\right) \theta_{1, \mu},
$$

where $\alpha_{h_{\mu}}$ and $\beta_{h_{\mu}}$ are as in (8) and (9), $\left(h_{\mu}\right)_{\theta}$ is as in (10), and $E_{2}^{(2)}(\tau):=$ $E_{2}(2 \tau)-\frac{1}{2} E_{2}(\tau) \in M_{2}\left(\Gamma_{0}(2)\right)$ and $E_{2}^{(4)}(\tau):=E_{2}(4 \tau)-\frac{1}{4} E_{2}(\tau) \in M_{2}\left(\Gamma_{0}(4)\right)$.

Proof. One can proceed exactly as in Atkinson [2]. More precisely, one can modify the proof of Theorem 3 in 2] to determine the action of the Ramanujan theta operator on $h_{\mu}$ in Equation (77). For brevity, we omit the detailed proof and only state the final result:

$$
\frac{\Theta\left(h_{\mu}\right)}{h_{\mu}}(\tau)=-\left(h_{\mu}\right)_{\theta}(\tau)-\alpha_{h_{\mu}} E_{2}(2 \tau)-\beta_{h_{\mu}} E_{2}(4 \tau)-\gamma_{h_{\mu}} E_{2}(\tau),
$$

where $\gamma_{h_{0}}:=\frac{1}{6}\left(k-\frac{1}{2}\right)-\frac{1}{3} N_{0}-\frac{2}{3} M$ and $\gamma_{h_{1}}:=\frac{1}{6}\left(k-\frac{1}{2}\right)-\frac{1}{3} N_{1}-\frac{2}{3} M$. Now, $L\left(\theta_{1, \mu}(\tau, z)\right)=0$, and hence

$$
L(\phi(\tau, z))=4 \Theta\left(h_{0}(\tau)\right) \theta_{1,0}(\tau, z)+4 \Theta\left(h_{1}(\tau)\right) \theta_{1,1}(\tau, z) .
$$

Thus Equations (12) and (13) imply Equation (11).

Remark. Choie and Kohnen [6] (following 3]) give an explicit characterization of $\frac{z \frac{\partial}{\partial z} \phi(\tau, z)}{\phi(\tau, z)}\left(\phi \in J_{k, m}\right)$, which also involves the "quasimodular" Eisenstein series $E_{2}$. However, our discussion of the action of the heat operator on Jacobi forms seems quite unrelated to the results in [6].

\section{Congruences And Reduction mod $p$}

For the remainder of our work, let $p \geq 5$ be a prime and (for simplicity) assume throughout that $k$ is even. For Jacobi forms $\phi(\tau, z)=\sum c(n, r) q^{n} \zeta^{r}$ and $\psi(\tau, z)=$ $\sum c^{\prime}(n, r) q^{n} \zeta^{r}$ with $p$-integral rational coefficients, we write $\phi \equiv \psi(\bmod p)$ when $c(n, r) \equiv c^{\prime}(n, r)(\bmod p)$ for all $n, r$. Recall the following fundamental fact regarding congruences of Jacobi forms.

Proposition 1 (Sofer [16]). Let $\phi(\tau, z) \in J_{k, m} \cap \mathbb{Z}[[q, \zeta]]$ and $\psi(\tau, z) \in J_{k^{\prime}, m^{\prime}} \cap$ $\mathbb{Z}[[q, \zeta]]$ such that $0 \not \equiv \phi \equiv \psi(\bmod p)$. Then $k \equiv k^{\prime}(\bmod p-1)$ and $m=m^{\prime}$.

Since there are congruences among Jacobi forms of different weights, it is desirable to find the smallest weight in which the (coefficient-wise) reduction of a Jacobi form modulo $p$ exists.

Definition 2. Let $\widetilde{M}_{k}:=\left\{f(\bmod p): f(\tau) \in M_{k} \cap \mathbb{Z}[[q]]\right\}$. Similarly, let $\widetilde{J}_{k, m}:=$ $\left\{\phi(\bmod p): \phi(\tau, z) \in J_{k, m} \cap \mathbb{Z}[[q, \zeta]]\right\}$. For modular forms and Jacobi forms with $p$-integral rational coefficients, we define the filtrations modulo $p$ by

$$
\omega(f):=\inf \left\{k: f(\bmod p) \in \widetilde{M}_{k}\right\}
$$

and

$$
\Omega(\phi):=\inf \left\{k: \phi(\bmod p) \in \widetilde{J}_{k, m}\right\}
$$


Serre [15] and Swinnerton-Dyer [17] thoroughly investigate filtrations of modular forms. Ono [13] gives a good overview of this theory and, in addition, discusses many interesting applications. Several of these applications rely on the crucial fact that if $f(\tau) \in M_{k} \cap \mathbb{Z}[[q]]$, then $\omega(\Theta(f)) \leq \omega(f)+p+1$, with equality if and only if $p \nmid \omega(f)$ (see Lemma 5 of [17). Our next proposition gives an analogous result for Jacobi forms.

Proposition 2. If $\phi(\tau, z) \in J_{k, m} \cap \mathbb{Z}[[q, \zeta]]$, then $L_{m}(\phi)(\bmod p)$ is the reduction of a Jacobi form modulo $p$. Moreover, we have

$$
\Omega\left(L_{m}(\phi)\right) \leq \Omega(\phi)+p+1,
$$

with equality if and only if $p \nmid(2 \Omega(\phi)-1) m$.

Proof. Note that $E_{p-1} \equiv 1(\bmod p)$ and $E_{2} \equiv E_{p+1}(\bmod p)$. If $\Omega(\phi)=k^{\prime}<k$, then $k-k^{\prime}=l(p-1)$ by Proposition 1 and $\phi \equiv\left(E_{p-1}\right)^{l} \psi(\bmod p)$, where $\psi \in$ $J_{k^{\prime}, m}$ with $\Omega(\psi)=k^{\prime}$. We find that $L_{m}(\phi) \equiv L_{m}(\psi)(\bmod p)$, i.e., $\Omega\left(L_{m}(\phi)\right)=$ $\Omega\left(L_{m}(\psi)\right)$, and hence we may (and do) assume that $\Omega(\phi)=k$. Equation (3) shows that $L_{m}(\phi)(\bmod p) \in \widetilde{J}_{k+p+1, m}$, i.e., $\Omega\left(L_{m}(\phi)\right) \leq k+p+1$.

If $p$ divides $(2 k-1) m$, then $\Omega\left(L_{m}(\phi)\right) \leq k+2<k+p+1$ by Equation (3). On the other hand, if $\Omega\left(L_{m}(\phi)\right)<k+p+1$, then $\Omega\left(\frac{(2 k-1) m}{6} \phi E_{2}\right)<k+p+1$ by Equation (3). It remains to show that $\Omega\left(\phi E_{2}\right)=k+p+1$, which then implies that $p$ divides $(2 k-1) m$. Recall that every Jacobi form (of even weight) can be written as

$$
\phi=\sum_{j=0}^{m} f_{j}\left(\phi_{-2,1}\right)^{j}\left(\phi_{0,1}\right)^{m-j},
$$

where $\phi_{-2,1}(\tau, z) \in \mathbb{Z}[[q, \zeta]]$ and $\phi_{0,1}(\tau, z) \in \mathbb{Z}[[q, \zeta]]$ are weak Jacobi forms of index 1 and weights -2 and 0 , respectively, and where each $f_{j} \in M_{k+2 j}$ has $p$-integral rational coefficients and is uniquely determined (see $\S 8$ and $\S 9$ of $[7$ for details and also for the corresponding result for Jacobi forms of odd weight). Moreover, there exists an $f_{j}$ such that $\omega\left(f_{j}\right)=k+2 j$, since otherwise $\Omega(\phi)<k$. Hence (using Theorem 2 and Lemma 5 of [17]), $\omega\left(f_{j} E_{2}\right)=k+2 j+p+1$, and we find that (by Lemma 2.2 of [16] $\Omega\left(\phi E_{2}\right)=k+p+1$, which completes the proof.

Let us introduce an analog of Atkin's $U$-operator for Jacobi forms:

Definition 3. For $\phi(\tau, z)=\sum_{\substack{n, r \\ 4 n m-r^{2} \geq 0}} c(n, r) q^{n} \zeta^{r} \in J_{k, m}$, we define

$$
\phi(\tau, z) \mid U_{p}:=\sum_{\substack{n, r \\ 4 n m-r^{2} \geq 0 \\ p \mid\left(4 n m-r^{2}\right)}} c(n, r) q^{n} \zeta^{r} .
$$

Remark. Note that the operator in Definition 3 is different from the operator $U_{l}$ in $\S 4$ of [7].

Our following result generalizes Proposition 6.3 of [1] and provides a useful tool in studying congruences defined by the action of the operator $U_{p}$. 
Proposition 3. Let $\phi(\tau, z)=\sum_{\substack{n, r \\ 4 n m-r^{2} \geq 0 \\ n \geq n_{0}}} c(n, r) q^{n} \zeta^{r} \in J_{k, m}^{\text {cusp }} \cap \mathbb{Z}[[q, \zeta]]$ such that $p \nmid m n_{0} c\left(n_{0}, 0\right)$. Then for every $s \in \mathbb{N}$, we have $\Omega\left(L_{m}^{s}(\phi)\right) \geq 12 n_{0}-2 m$.

Proof. To see that $k \geq 12 n_{0}-2 m$, apply the valence formula to the cusp form $\phi(\tau, 0) \in M_{k}$ or to some $f_{j} \not \equiv 0$ in the decomposition (15) of $\phi$ if $\phi(\tau, 0) \equiv 0$ (in which case $f_{0}(\tau) \equiv 0$, but $f_{j}(\tau) \not \equiv 0$ for some $\left.j \neq 0\right)$. Let $\Delta(\tau):=q-24 q^{2}+\ldots$ be the usual cusp form of weight 12. The Ramanujan identities (2) imply that $\Theta(\Delta)=\Delta E_{2}$, and a straightforward computation shows that

$$
\frac{L_{m}^{s}(\phi)(\tau, z)}{\Delta^{n_{0}}(\tau)}=\left(4 m n_{0}\right)^{s} c\left(n_{0}, 0\right)+\sum_{(n, r) \neq(0,0)} C(n, r) q^{n} \zeta^{r},
$$

where $C(n, r) \in \mathbb{Z}$. In particular, $\Delta^{-n_{0}} L_{m}^{s}(\phi)$ is congruent modulo $p$ to a weak Jacobi form $\psi \not \equiv 0(\bmod p)$. Note that the weight of a nonzero weak Jacobi form is at least $-2 m$ and hence $\Omega\left(L_{m}^{s}(\phi)\right) \geq 12 n_{0}-2 m$.

As an application of Propositions 2 and 3, we now determine when $U_{p}$ applied to a Jacobi form is nonzero modulo $p$. Our result is of the same flavor as Theorem 1.7 of Ahlgren and Ono [1] (see Elkies, Ono, and Yang [8] and Guerzhoy [10] for general discussions of congruences that involve Atkin's $U$-operator).

Theorem 3. Let $\phi(\tau, z)=\sum_{\substack{n, r \\ 4 n m-r^{2} \geq 0 \\ n \geq n_{0}}} c(n, r) q^{n} \zeta^{r} \in J_{k, m}^{c u s p} \cap \mathbb{Z}[[q, \zeta]]$. Suppose that $p \geq \max \left(5, k-12 n_{0}+2 m\right)$ is a prime such that $p \nmid m n_{0} c\left(n_{0}, 0\right)$. If $2 \Omega\left(L_{m}(\phi)\right) \equiv$ $3,5(\bmod p)$, then $\phi \mid U_{p} \not \equiv 0(\bmod p)$.

Proof. We proceed as in [1]. Suppose that $\phi \mid U_{p} \equiv 0(\bmod p)$. Note that $\phi \mid U_{p} \equiv$ $\phi-L_{m}^{p-1}(\phi)(\bmod p)$ for any $\phi$ and hence

$$
0 \not \equiv L_{m}^{p-1}(\phi) \equiv \phi(\bmod p) .
$$

If $p \nmid\left(2 \Omega\left(L_{m}^{p-2}(\phi)\right)-1\right)$, then $\Omega\left(L_{m}^{p-1}(\phi)\right)=\Omega\left(L_{m}^{p-2}(\phi)\right)+p+1$ by Proposition [2. However, Equation (17) shows that $\Omega\left(L_{m}^{p-1}(\phi)\right) \leq k$ while Proposition 3 gives $\Omega\left(L_{m}^{p-2}(\phi)\right) \geq 12 n_{0}-2 m$, which contradicts the assumption that $p \geq k-12 n_{0}+2 m$.

Consequently, we have that $p$ divides $\left(2 \Omega\left(L_{m}^{p-2}(\phi)\right)-1\right)$. Let $j \leq p-3$ be the smallest positive integer such that $p$ divides $\left(2 \Omega\left(L_{m}^{j+1}(\phi)\right)-1\right)$. Proposition 2 implies that

$$
2 \Omega\left(L_{m}^{j+1}(\phi)\right)=2\left(\Omega\left(L_{m}(\phi)\right)+j(p+1)\right) \equiv 2 \Omega\left(L_{m}(\phi)\right)+2 j \equiv 1(\bmod p),
$$

which contradicts the assumption that $2 \Omega\left(L_{m}(\phi)\right) \equiv 3,5(\bmod p)$. We conclude that $\phi \mid U_{p} \not \equiv 0(\bmod p)$.

For example, if

$$
\phi_{10,1}(\tau, z)=\left(\zeta-2+\zeta^{-1}\right) q+\ldots \in J_{10,1}^{\text {cusp }} \cap \mathbb{Z}[[q, \zeta]]
$$

and $p=17$, then $2 \Omega\left(L\left(\phi_{10,1}\right)\right)=56 \equiv 5(\bmod 17)$ and Theorem 3 implies that $\phi_{10,1} \mid U_{17} \not \equiv 0(\bmod 17)$. Similarly, if

$$
\phi_{12,1}(\tau, z)=\left(\zeta+10+\zeta^{-1}\right) q+\ldots \in J_{12,1}^{\text {cusp }} \cap \mathbb{Z}[[q, \zeta]]
$$


and $p=7$, then $2 \Omega\left(L\left(\phi_{12,1}\right)\right)=40 \equiv 5(\bmod 7)$ and Theorem 3 implies that $\phi_{12,1} \mid U_{7} \not \equiv 0(\bmod 7)$.

\section{ACKNOWLEDGMENT}

The author thanks Kathrin Bringmann for several useful discussions.

\section{REFERENCES}

[1] Ahlgren, S., and Ono, K. Arithmetic of singular moduli and class polynomials. Compos. Math. 141, no. 2 (2005), 293-312. MR2134268(2006a:11058)

[2] Atkinson, J. Divisors of modular forms on $\Gamma_{0}(4)$. J. Number Theory 112, no. 1 (2005), 189 204. MR2131143 (2005m:11084)

[3] Bruinier, J., Ono, K., and Kohnen, W. The arithmetic of the values of modular functions and the divisors of modular forms. Compos. Math. 140, no. 3 (2004), 552-566. MR2041768 (2005h:11083)

[4] Choie, Y. Jacobi forms and the heat operator. Math. Z. 225 (1997), 95-101. MR.1451334 (98c:11042)

[5] Choie, Y. Jacobi forms and the heat operator II. Illinois J. Math. 42, no. 2 (1998), 179-186. MR1612731 (99d:11049)

[6] Choie, Y., and Kohnen, W. Special values of elliptic functions at points of the divisors of Jacobi forms. Proc. Amer. Math. Soc. 131, no. 11 (2003), 3309-3317. MR.1990618 (2004e:11042)

[7] Eichler, M., and Zagier, D. The theory of Jacobi forms. Birkhäuser, Boston, 1985. MR781735 (86j:11043)

[8] Elkies, N., Ono, K., and Yang, T. Reduction of CM elliptic curves and modular function congruences. Internat. Math. Res. Notices 2005, no. 44, 2695-2707. MR2181309(2006k:11076)

[9] Guerzhoy, P. An approach to the p-adic theory of Jacobi forms. Internat. Math. Res. Notices 1994, no. 1, 31-39. MR.1255251 (94m:11057)

[10] Guerzhoy, P. On U(p)-congruences. Proc. Amer. Math. Soc. 135, no. 9 (2007), 2743-2746. MR.2317947

[11] Kaneko, M., and Zagier, D. A generalized Jacobi theta function and quasimodular forms, The moduli space of curves (Texel Island, 1994). Progr. Math. 129. Birkhäuser, 1995, pp. 165-172. MR:1363056 (96m:11030)

[12] Kawai, T., and Yoshioka, K. String partition functions and infinite products. Adv. Theor. Math. Phys. 4, no. 2 (2000), 397-485. MR1838446 (2002g:11054)

[13] Ono, K. The web of modularity: Arithmetic of the coefficients of modular forms and q-series, vol. 102 of CBMS Regional Conference Series in Mathematics. Published for the Conference Board of the Mathematical Sciences, Washington, DC, by the Amer. Math. Soc., Providence, RI, 2004. MR2020489 (2005c:11053)

[14] Ramanujan, S. On certain arithmetical functions. Trans. Camb. Phil. Soc. 22 (1916), 159-184 (Collected Papers, No. 18).

[15] Serre, J-P. Formes modulaires et fonctions zeta p-adiques, Modular functions of one variable, III. Lecture Notes in Math., 350. Springer, 1973, pp. 191-268. MR0404145 (53:7949a)

[16] Sofer, A. p-adic aspects of Jacobi forms. J. Number Theory 63, no. 2 (1997), 191-202. MR:1443756 (98b:11058)

[17] Swinnerton-Dyer, H. P. F. On l-adic representations and congruences for coefficients of modular forms, Modular functions of one variable, III. Lecture Notes in Math., 350. Springer, 1973, pp. 1-55. MR0406931(53:10717a)

Department of Mathematics, University of North Texas, Denton, Texas 76203

E-mail address: richter@unt.edu 